\title{
Anti-metastatic activity of fangchinoline in human gastric cancer AGS cells
}

\author{
ZHENGRONG CHEN, TENGFEI HE, KUI ZHAO and CHUNGEN XING
}

Department of General Surgery, The Second Affiliated Hospital of Soochow University, Suzhou, Jiangsu 215007, P.R. China

Received April 29, 2015; Accepted November 10, 2016

DOI: $10.3892 / 01.2016 .5457$

\begin{abstract}
Fangchinoline (FCL) is an active component isolated from the traditional medicinal plant Stephania tetrandra S. Moore, and has been reported to possess anti-cancer functions in several types of cancers; however, the effect of FCL on gastric cancer metastasis and its underlying molecular mechanisms remain unknown. The current study aimed to investigate the effect of FCL on the cell migration and invasion of human metastatic gastric cancer AGS cells and its mechanisms. Our study demonstrates that FCL dosage dependently suppressed the adhesion, migration and invasion capacities of human gastric cancer AGS cells without obvious cytotoxic effects. Reverse transcription-polymerase chain reaction and western blot assays demonstrated that FCL greatly inhibited the expression of matrix metalloproteinase (MMP)-2 and MMP-9 at both the mRNA and protein levels, while it significantly increased the expression of tissue inhibitor of metalloproteinase (TIMP) 1 and TIMP2 messenger RNAs. Our results also indicated that FCL repressed the phosphorylation of AKT in gastric cancer AGS cells. In summary, FCL may exert its anti-metastatic property in human gastric cancer cells in vitro by suppression of MMP-2 and MMP-9, increase of TIMP1 and TIMP2 genes, and inhibition of AKT phosphorylation. FCL may be a drug candidate for the treatment of gastric cancer metastasis.
\end{abstract}

\section{Introduction}

Gastric cancer is the fourth most common neoplasia and the second highest cause of mortality associated with cancer worldwide (1). It is reported that gastric cancer is also the third most common type of cancer and the second highest cause of mortality in patients with cancer in China (1). Complete excision of the gastric tumor is the best option of curative treatment (2). In

Correspondence to: Professor Chungen Xing, Department of General Surgery, The Second Affiliated Hospital of Soochow University, Building 3, 1055 Sanxiang Road, Suzhou, Jiangsu 215007, P.R. China

E-mail: xingcgsz@163.com

Key words: fangchinoline, gastric cancer, metastasis, MMPs, AKT patients diagnosed at advanced stages, although chemotherapy effectively improves the survival quality and time, the median survival time of patients with advanced gastric cancer remains poor $(1,2)$. The metastatic ability of gastric cancer is the leading cause of poor prognosis and high mortality (3). Therefore, cancer metastasis is still a big challenge in the clinic for oncologists.

Accumulating data have demonstrated that degradation of extracellular matrix (ECM) proteins is the antecedent condition of malignant tumor metastasis (1). Matrix metalloproteinases (MMPs) are part of the endopeptidase family and can cleave the majority of components of the ECM, including fibronectin, collagen, elastin, proteoglycan and laminin, thus playing critical roles in the development, progression and metastasis of malignant tumors $(3,4)$. Upregulation of MMPs and altered expression of their tissue inhibitors [known as tissue inhibitors of metalloproteinases (TIMPs)] are associated with the invasiveness and metastasis of cancer cells (4). Among them, MMP-2 and MMP-9 are the main enzymes for the degradation of basement membranes in cancer cells and/or stromal cells $(4,5)$. TIMPs are natural inhibitors of MMPs, and TIMP2 specifically inhibits MMP-2, while TIMP1 inhibits MMP-9 (3).

Fangchinoline (FCL) is a bisbenzylisoquinoline alkaloid in Stephania tetrandra S. Moore (Fen fang ji) (6). It is reported that FCL can inhibit histamine release (7), lower blood pressure as a non-specific calcium channel antagonist (8) and inhibit glutamate release from rat cortical synaptosomes (9). In addition, FCL exerts anti-cancer activities in several types of malignant tumors, including breast cancer (10), prostate carcinoma (11), hepatocellular carcinoma (12) and lung cancer (13). However, the effects of FCL on the metastasis of gastric cancer and its underlying mechanisms remain poorly understood.

In the present study, the anti-metastatic activity of FCL (Fig. 1) in gastric cancer cells and its molecular mechanism of action were explored. Our data revealed that FCL inhibited the phosphorylation of AKT and upregulated TIMP2/1, leading to reduced expression of MMP-2/9 and inhibition of gastric cancer cell invasion in vitro.

\section{Materials and methods}

Chemicals and antibodies. FCL (purity >98\%) was purchased from Shanghai Standard Technology Co., Ltd. (Shanghai, China). Cell culture materials were purchased from Gibco (Thermo Fisher Scientific, Inc., Waltham, MA, USA). Specific 
antibodies against GAPDH (1:1,000, sc-25778), MMP-2 (1:500, sc-53630), MMP-9 (1:500, sc-21733), phosphorylated (p)-AKT (1:500, sc-135650) and AKT (1:500, sc-5298) were purchased from Santa Cruz Biotechnology, Inc. (Dallas, TX, USA).

Cell culture. Human gastric cancer AGS cells (Type Culture Collection of the Chinese Academy of Sciences, Shanghai, China) were cultured in high glucose-Dulbecco's modified Eagle's medium (DMEM) containing 10\% fetal bovine serum (FBS; both HyClone; GE Healthcare Life Sciences, Chalfont, UK) and $200 \mathrm{mM}$ glutamine at $37^{\circ} \mathrm{C}$ under a humidified $95 \%$ air $/ 5 \% \mathrm{CO}_{2}$ mixture $(\mathrm{v} / \mathrm{v})$.

Cell viability assay. Cells were seeded at a density of $5 \times 10^{3}$ cells/well in a 96-well microplate containing DMEM with $10 \%$ FBS, and incubated for $24 \mathrm{~h}$. Next, cells were incubated with different concentrations of FCL for $24 \mathrm{~h}$. The proliferation rate was determined with an MTT assay kit (Beyotime Institute of Biotechnology, Shanghai, China). The results were expressed as the absorbance at $570 \mathrm{~nm}$, as previously reported (11).

Cell adhesion assay. After pre-treating the cells with or without FCL $(2,4$ and $8 \mu \mathrm{M})$ for $24 \mathrm{~h}$, AGS cells were trypsinized and suspended at a final concentration of $2 \times 10^{5}$ cells $/ \mathrm{ml}$ in serum-free medium. A total of $100 \mu \mathrm{l}$ cell suspension was seeded to each well in a 96-well plate coated with $20 \mu \mathrm{g} / \mathrm{ml}$ fibronectin overnight. The cells were incubated for $30 \mathrm{~min}$ at $37^{\circ} \mathrm{C}$, and the non-adherent cells were removed by washing with PBS. MTT assay was used to determine the number of remaining adherent cells.

Wound healing assay. AGS cells were seeded on a 6-well plate and incubated in serum-free medium for $24 \mathrm{~h}$. A transverse scratch wound on each monolayer of cells was created using a sterilized $200-\mu 1$ pipette tip. The scratch wounded cell monolayers were then stimulated with various concentrations of FCL $(0,2,4$ and $8 \mu \mathrm{M})$ in $5 \%$ FBS for an additional $36 \mathrm{~h}$, at which point, the cells that had migrated into the wound were photographed and analyzed.

Transwell chamber invasion assay. Transwell chamber invasion assay was conducted as described by Magee et al (14). In brief, the transwell chambers (EMD Millipore, Billerica, MA, USA) were pre-coated with Matrigel (BD Biosciences, Franklin Lakes, NJ, USA) for $1 \mathrm{~h}$, and the upper chambers were washed using serum-free medium. Following incubation of AGS cells with FCL $(0,2,4$ and $8 \mu \mathrm{M})$ for $24 \mathrm{~h}$, the cells were trypsinized and suspended at a density of $2 \times 10^{5}$ cells $/ \mathrm{ml}$ in serum-free medium. A total of $200 \mu \mathrm{l}$ cells were placed in the upper chambers, and medium with $10 \%$ FBS was placed in the lower chambers. After $24-\mathrm{h}$ incubation at $37^{\circ} \mathrm{C}$, the non-invaded cells were removed using a cotton swab, while the invaded cells were fixed with $100 \%$ methanol and stained with hematoxylin and eosin (Nanjing Jiancheng Biotechnology Institute Co., Ltd., Nanjing, China). The invaded cells on the lower surface of the membrane filter were counted under an inverted microscope. The data are presented as the mean number of cells attached to the bottom surface from six randomly selected fields.
RNA extraction and reverse transcription-polymerase chain reaction (RT-PCR). Total RNA was isolated from AGS cells using TRIzol reagent (Invitrogen; Thermo Fisher Scientific, Inc.), according to the manufacturer's protocol. Synthesis of complementary DNA was performed using the PrimeScript RT Reagent kit (Takara Biotechnology Co., Ltd., Dalian, China), and the PCR conditions were as follows: Initial denaturation at $95^{\circ} \mathrm{C}$ for $5 \mathrm{~min}$, followed by $24-33$ cycles of denaturation at $94^{\circ} \mathrm{C}$ for $30 \mathrm{sec}$, annealing at $54^{\circ} \mathrm{C}$ for $30 \mathrm{sec}$ and extension at $72^{\circ} \mathrm{C}$ for $45 \mathrm{sec}$. The primer sequences were as follows: MMP-2 (forward 5'-TGGATGATGCCTTTGCTCGT-3' and reverse 5'-AAACTT GCAGGGCTGTCCTT-3'); MMP-9 (forward 5'-GGACAA GCTCTTCGGCTTCT-3' and reverse 5'-TTCAGGGCGAGG ACCATAGA-3'); TIMP1 (forward 5'-CTCGTCATCAGGGCC AAGTT-3' and reverse 5'-GTAGGTCTTGGTGAAGCCCC-3'); TIMP2 (forward 5'-TAGTGATCAGGGCCAAAGCG-3' and reverse 5'-CAGGCTCTTCTTCTGGGTGG-3'); and GAPDH (forward 5'-GAGAAGGCTGGGGCTCATTT-3' and reverse 5'-GTCAGGTCCACCACTGACAC-3'). The expression levels of MMP-2, MMP-9, TIMP1 and TIMP2 were normalized to those of GAPDH, which served as an internal control.

Western blot analysis. To analyze the level of protein expression, cell lysates were prepared, as described previously (15), and separated by $10 \%$ SDS-PAGE and then transferred to polyvinylidene difluoride membranes for $2 \mathrm{~h}$. The membrane was treated with blocking solution (5\% bovine serum albumin in TBS) at room temperature for $1 \mathrm{~h}$, and then incubated overnight at $4^{\circ} \mathrm{C}$ with antibodies against MMP-2, MMP-9, AKT, p-AKT or GAPDH. The membranes were washed with Tris-buffered saline containing 0.5\% Tween-20 (Beyotime Institute of Biotechnology), and then incubated for $1 \mathrm{~h}$ at room temperature with an anti-immunoglobulin G secondary antibody conjugated to horseradish peroxidase (1:1,000; A0208; Beyotime Institute of Biotechnology). The expression levels of the proteins were analyzed via chemiluminescence (Beyotime Institute of Biotechnology) and quantified using Quantity One software, version 4.6.2 (Bio-Rad Laboratories, Inc., Hercules, CA, USA). The expression levels of MMP-2, MMP-9, p-AKT and AKT were normalized to those of the internal control GAPDH.

Statistical analysis. The results are expressed as the mean \pm standard deviation from $\geq 3$ independent experiments. The statistical differences between the experimental groups were assessed using a Student's $t$-test in SPSS version 13.0 (SPSS Inc., Chicago, IL, USA). $\mathrm{P}<0.05$ was considered to indicate a statistically significant difference.

\section{Results}

FCL decreases cell proliferation in gastric cancer AGS cells. To determine the inhibitory effect of FCL (Fig. 1A) on gastric cancer cell proliferation, an MTT assay was used. AGS cells were pre-treated with FCL $(0-64 \mu \mathrm{M})$ for $24 \mathrm{~h}$. As shown in Fig. 1B, gastric cancer cell proliferation was inhibited by FCL in a dose-dependent manner, while FCL at doses $<8 \mu \mathrm{M}$ exhibited little effect on the cell viability of gastric cancer AGS cells (Fig. 1B). Therefore, these doses $(0,2,4$ and $8 \mu \mathrm{M})$ were used in the following experiments. 
A

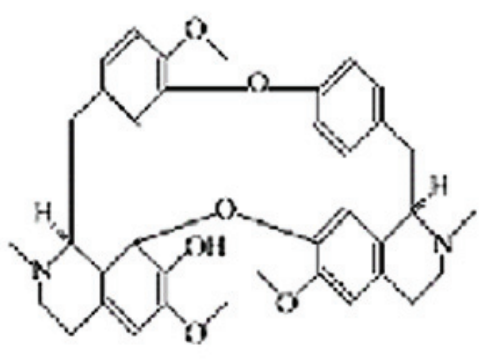

B

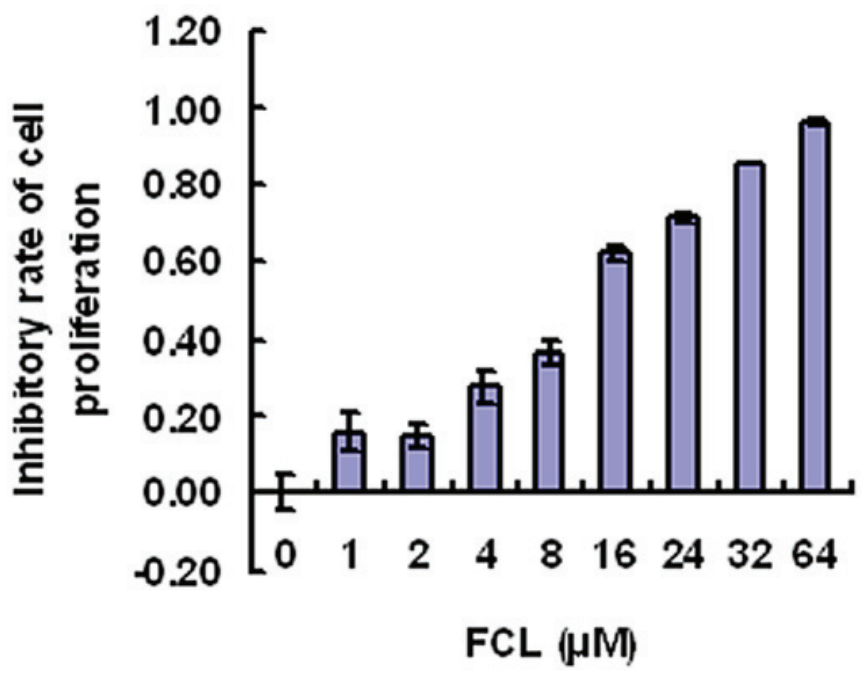

Figure 1. Effect of FCL on cell proliferation in human gastric cancer AGS cells. (A) Chemical structure of FCL. (B) AGS cells were seeded in a 96-well plate and incubated with the indicated concentrations of FCL for $24 \mathrm{~h}$. MTT assay was then performed to assess cell viability. The graph indicates the representative cell viability from three independent experiments. Data are presented as the mean \pm standard deviation. FCL, fangchinoline.

FCL inhibits the attachment of gastric cancer cells to fibronectin. To determine the effect of FCL on cell adhesion to the ECM, an adhesion assay was conducted in gastric cancer AGS cells. As shown in Fig. 2, FCL greatly inhibited cell adhesion ability in a dose-dependent manner, as demonstrated by cell attachment assay. Cell adhesion was inhibited by $41.53 \pm 4.85 \%$ for the $4-\mu \mathrm{M}$ dose of FCL and by $85.63 \pm 3.51 \%$ for $8 \mu \mathrm{M}$ FCL (Fig. 2).

$F C L$ reduces the migration of gastric cancer cells. Next, the inhibitory effect of FCL on cell migration was assessed in the artificial scratch wound of gastric cancer AGS cells. As shown in Fig. 3A, FCL obviously reduced cell numbers in the scratch wound area in gastric cancer AGS cells in a dose-dependent manner. The inhibitory rates of cell migration were $29.55 \pm 5.74$, $64.65 \pm 4.99$ and $76.68 \pm 4.82 \%$ for 2,4 and $8 \mu \mathrm{M} \mathrm{FCL}$, respectively (Fig. 3B).

FCL suppresses cell invasion in gastric cancer cells. A Boyden chamber assay was used to examine whether FCL could suppress the cell invasion of gastric cancer cells. As shown in Fig. 4A, exposure to FCL greatly decreased the invasive ability of gastric cancer cells in a concentration-dependent manner. The inhibitory rates of FCL on gastric cancer cells invasion were $13.47 \pm 7.30,51.93 \pm 3.56$ and $75.97 \pm 4.30 \%$ for 2 , 4 and $8 \mu \mathrm{M}$ FCL, respectively (Fig. 4B).

Effects of FCL on the expression of MMP-2, MMP-9, TIMP2 and TIMP1 genes. Accumulating evidence has demonstrated that MMPs, particularly MMP-2/MMP-9, and their inhibitors TIMP2/TIMP1 participated in the invasiveness and metastasis of malignant tumors via stimulating the degradation of ECM and cell migration $(3,5)$. To verify the possible anti-metastatic molecular mechanism of FCL on gastric cancer cells, the expression of MMP-2/MMP-9 and TIMP2/TIMP1 genes was detected in FCL-treated gastric cancer AGS cells. The expression of MMP-2 and MMP-9 messenger (m) RNAs was obviously decreased in a concentration-dependent manner, with a maximum decrease

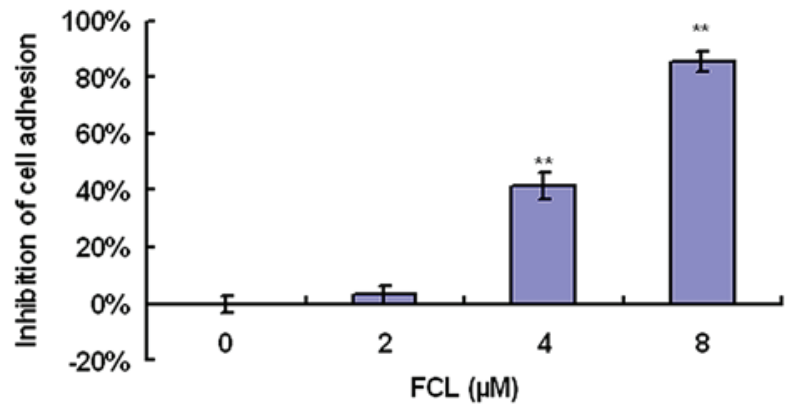

Figure 2. Inhibition of cell adhesion to fibronectin by FCL in gastric cancer AGS cells. Cells were treated with the indicated doses of FCL for $24 \mathrm{~h}$. Subsequently, the cells ( $2 \times 10^{4}$ cells/well) were added to 96 -well plates coated with fibronectin, and the plates were incubated for $30 \mathrm{~min}$. The medium was then carefully removed from each well, and each well was then washed three times with PBS. Next, MTT assay was used to determine the number of adherent cells. ${ }^{* *} \mathrm{P}<0.01$ compared with the control. Data are presented as the mean \pm standard deviation of three separate experiments. FCL, fangchinoline.

of $31.23 \pm 4.16$ and $63.83 \pm 4.64 \%$ for MMP-2 and MMP-9, respectively, following exposure to $8 \mu \mathrm{M}$ FCL (Fig. 5). In addition, the mRNA levels of their specific inhibitors TIMP2 and TIMP1 were significantly increased in a dose-dependent manner, with a maximum elevated rate of $1.96 \pm 0.08$-fold and $1.50 \pm 0.06$-fold for TIMP2 and TIMP1, respectively, upon exposure to $8 \mu \mathrm{M}$ FCL (Fig. 5). Additionally, the expression levels of MMP-2 and MMP-9 were also confirmed by western blot assay, and the results demonstrated that FCL also significantly inhibited the expression of MMP-2 and MMP-9 proteins in a similar pattern (Fig. 6). The above data suggested that FCL inhibited the invasiveness of gastric cancer AGS cells via downregulation of MMP-2/MMP-9 and upregulation of TIMP2/TIMP1.

FCL reduces the phosphorylation of AKT in gastric cancer cells. Since our data revealed that FCL exhibited inhibitory effects on cell adhesion, migration, invasion and proteinases, the effects of FCL on the expression of the components of the 
A
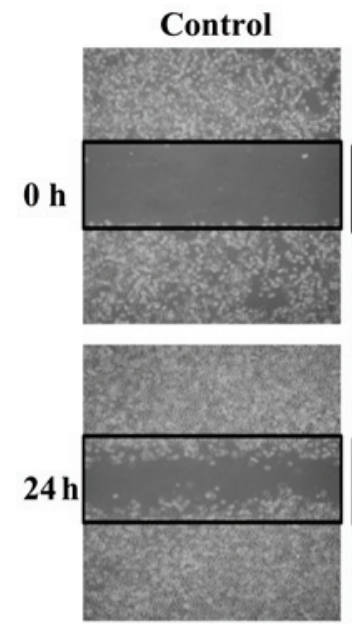

$2 \mu \mathbf{M}$
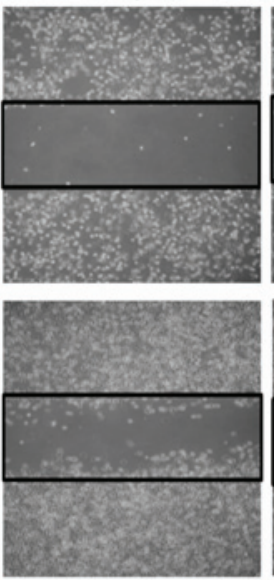

$4 \mu \mathrm{M}$
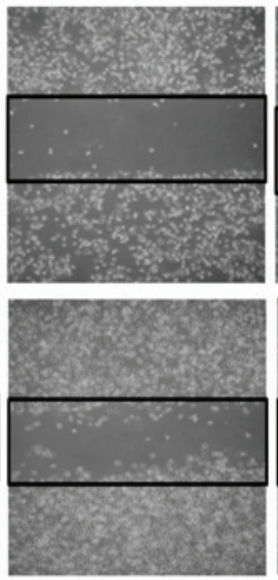

$8 \mu \mathrm{M}$
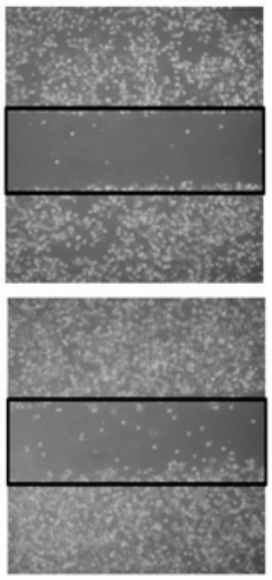

B

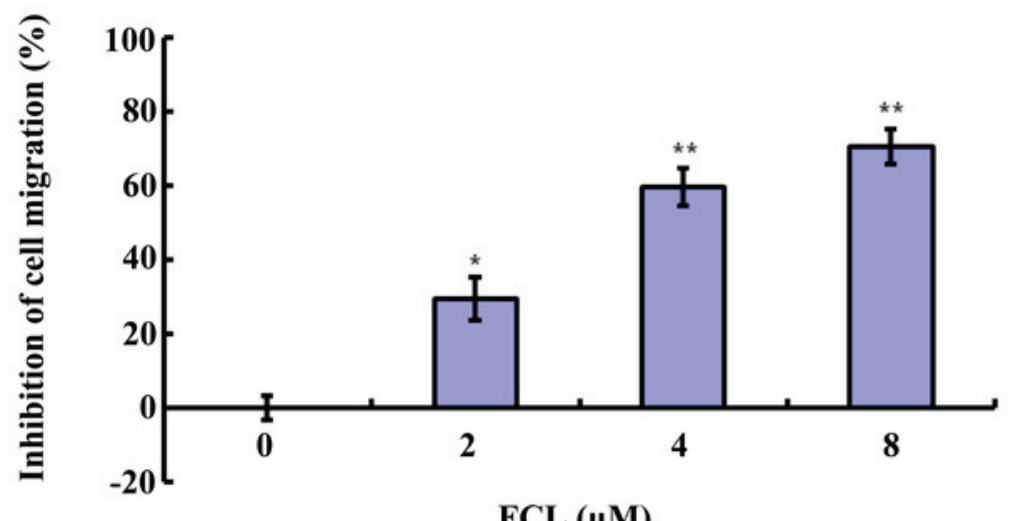

FCL $(\mu \mathrm{M})$

Figure 3. Suppression of cell motility by FCL in gastric cancer AGS cells. Cells were seeded in a 6-well plate and scratches were created using a 200- $\mu 1$ micropipette in confluent cultures. Subsequently, the cells were washed with serum-free medium and then treated with the indicated doses of FCL in medium containing 5\% fetal bovine serum, and allowed to grow. (A) At the indicated times, wound closures were photographed under a microscope (magnification, $\mathrm{x} 40$ ). (B) Migrated cell numbers within the black lines were counted and analyzed. ${ }^{*} \mathrm{P}<0.05,{ }^{* *} \mathrm{P}<0.01$ vs. the control. Data are presented as the mean \pm standard deviation of three separate experiments. FCL, fangchinoline.

A
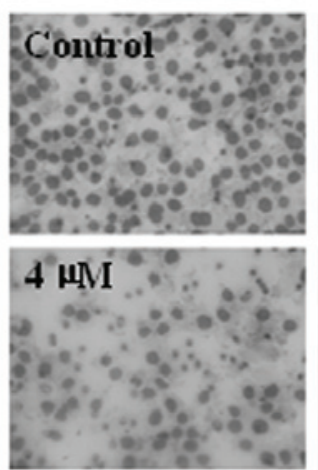
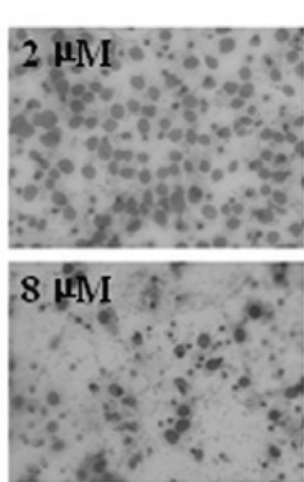

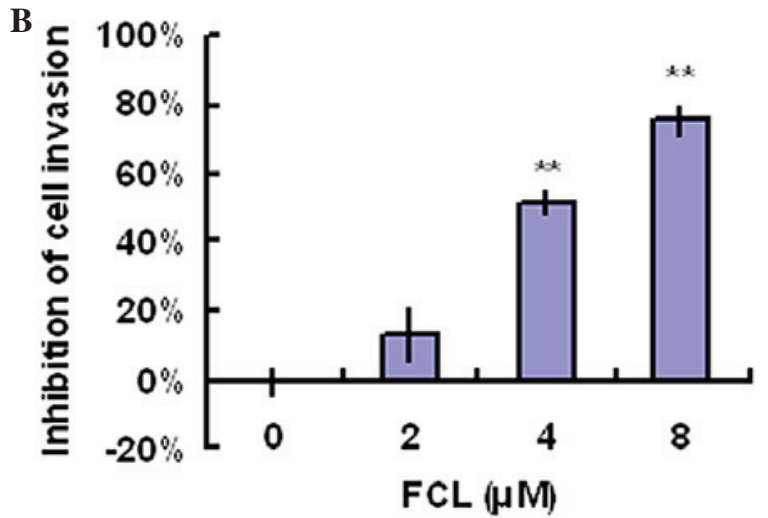

Figure 4. Inhibition of cell invasion by FCL in AGS cells. Cells were pre-treated with FCL for $24 \mathrm{~h}$, and the same number of cells was plated onto the upper chamber of a Transwell chamber coated with Matrigel in serum-free medium. Medium containing $10 \%$ fetal bovine serum was placed in the bottom chamber to act as a chemoattractant. Following $24 \mathrm{~h}$ of incubation, the membranes were wiped from the upper chamber with a small scalper blade, and stained with hematoxylin and eosin. (A) Upon fixing, the membrane was observed under an inverted microscope (magnification, x200). (B) The number of invaded cells was counted. ${ }^{* *} \mathrm{P}<0.01$ compared with the control. Data are presented as the mean \pm standard deviation of three separate experiments. FCL, fangchinoline.

phosphatidylinositol-4,5-bisphosphate 3-kinase (PI3K)/AKT pathway were explored by western blotting to elucidate the underlying molecular mechanisms. As shown in Fig. 6, the phosphorylation of AKT was significantly reduced in a dose-dependent manner to $79.6 \pm 4.2$ and $31.8 \pm 3.5 \%$ upon treatment with 4 and $8 \mu \mathrm{M}$ FCL, respectively, while the total AKT levels were not significantly affected upon exposure to FCL. 
A

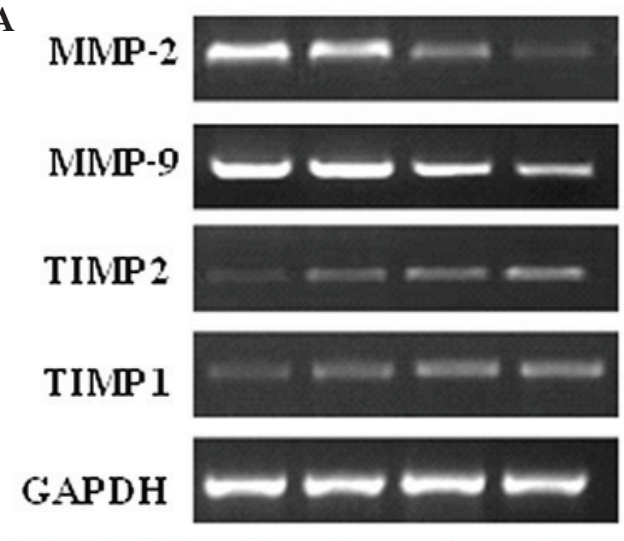

B

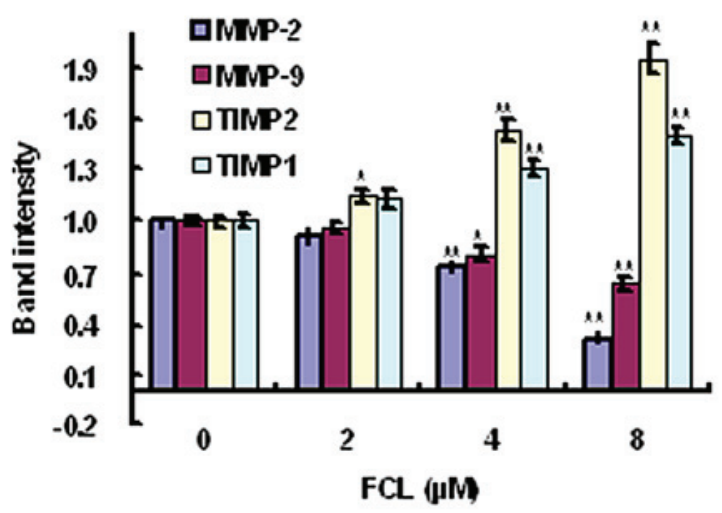

Figure 5. Effects of FCL on the expression of MMP-2, MMP-9, TIMP1 and TIMP2 mRNAs. (A) Gastric cancer cells were treated with or without FCL $(2-8 \mu \mathrm{M})$ for $24 \mathrm{~h}$. Total RNA was isolated, and reverse transcription-polymerase chain reaction was performed to detect the expression of MMP-2, MMP-9, TIMP1 and TIMP2 mRNAs. (B) The densitometry of the bands was normalized to the expression of GAPDH and analyzed. "P<0.05, ${ }^{* * *} \mathrm{P}<0.01$ compared with the control. Data are presented as the mean \pm standard deviation of three separate experiments. FCL, fangchinoline; mRNA, messenger RNA; MMP, matrix metalloproteinase; TIMP, tissue inhibitor of metalloproteinase.

A

C
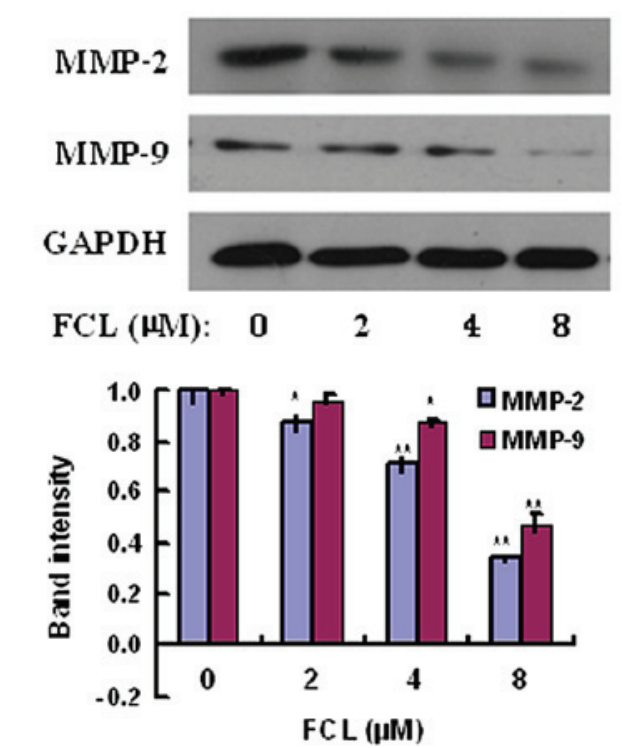

B

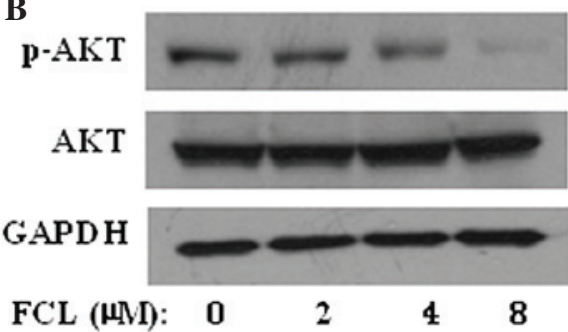

D

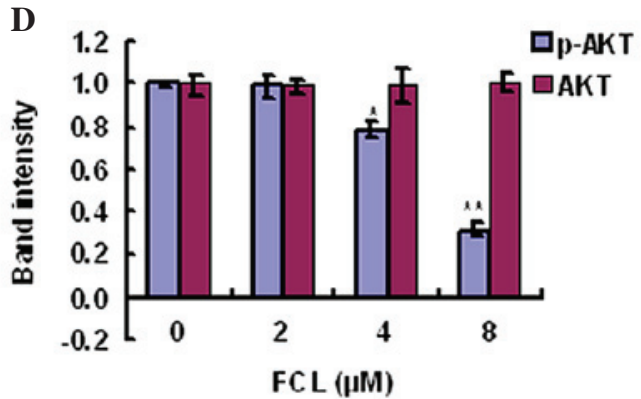

Figure 6. Effects of FCL on the expression of MMP-2/MMP-9 and phosphorylation of AKT in gastric cancer cells. (A) Cells were treated with or without FCL $(0-8 \mu \mathrm{M})$ for $24 \mathrm{~h}$. Total protein was extracted, and western blotting was performed to assess the expression of MMP-2 and MMP-9 proteins. (B) Western blotting was performed to assess the expression of p-AKT and AKT proteins. (C and D) The densitometry of the bands was normalized to the expression of GAPDH and analyzed. "P $<0.05,{ }^{* *} \mathrm{P}<0.01$ compared with the control. Data are presented as the mean \pm standard deviation of three separate experiments. FCL, fangchinoline; mRNA, messenger RNA; MMP, matrix metalloproteinase; p, phosphorylated.

\section{Discussion}

As an active compound of Stephania tetrandra S. Moore, FCL has been reported to exhibit anti-cancer effects, which are associated with cell apoptosis promotion, autophagic death induction, cell cycle arrest and multidrug resistance (6-8); however, the anti-metastatic activity of FCL has not been clarified yet, particularly in gastric cancer. The present study attempted to identify novel functions of FCL, and our data revealed that FCL inhibited cell adhesion, migration and invasion in human gastric cancer AGS cells without obvious cytotoxicity. In addition, the anti-metastatic function of FCL was associated with downregulation of MMP-2/9, upregulation of TIMP2/1 and decreased phosphorylation of AKT.
The invasion and metastasis of malignant tumors are the leading causes of poor prognosis in patients with gastric cancer, and these processes are usually accompanied by the destruction of basement membrane components in the ECM, which is mainly controlled by a number of proteolytic enzymes such as MMPs and their endogenous inhibitors TIMP2/1 $(1,5)$. Immunohistochemistry analysis revealed that expression of MMP-2 and MMP-9 was detected in 94 and 70\% of gastric cancer specimens, respectively, and it was associated with poor overall survival in patients with gastric cancer $(16,17)$. Overexpression of MMP-2/9 was observed to accelerate the invasion of tumor cells in vitro and in vivo $(18,19)$, and metastasis in MMP-2 and MMP-9-null mice was greatly suppressed compared with that in wild-type mice, suggesting that the elevated expression 
of MMP-2/9 promotes the metastatic potential of malignant tumor cells $(3,5)$. Therefore, suppression of cancer migration and invasion mediated by MMP-2/9 provides a new direction for drug research in cancer metastasis. In the current study, exposure of human gastric cancer AGS cells to non-toxic doses of FCL inhibited cell adhesion, migration and invasion, compared with control cells, in a concentration-dependent manner. In addition, FCL was observed to inhibit the expression of MMP-2/9 mRNAs as well as their protein levels, while it increased the expression of TIMP2/1 genes, implying that FCL exhibited an ability to inhibit matrix degradation proteases, thus limiting the adhesive and invasive capabilities of gastric cancer cells.

A considerable number of studies have shown that $\mathrm{PI} 3 \mathrm{~K} / \mathrm{AKT}$ signaling serves a critical role in regulating the expression of MMPs; therefore, suppression of PI3K/AKT signaling may inhibit proliferation, invasion, angiogenesis and metastasis in various malignant tumors (15). In addition, inhibition of PI3K/AKT signaling could result in decreased expression of MMP-2/9 (20-22). Considering that FCL at non-toxic doses $(0-8 \mu \mathrm{M})$ could greatly suppress cell adhesion, migration and invasion via inhibition of MMP-2/9 mRNAs and proteins, the present authors hypothesized whether $\mathrm{PI} 3 \mathrm{~K} / \mathrm{AKT}$ signaling was involved in this process. Our results demonstrated that FCL suppressed the phosphorylation of AKT in gastric cancer cells, suggesting that the inhibition of cell adhesion, migration and invasion by FCL could be mediated by suppression of MMP-2/9 and increase of TIMP2/1, in part, via decreasing the phosphorylation of AKT.

In summary, our current study has shown that FCL prevented the metastatic potential of human gastric cancer AGS cells via inhibition of MMP-2/9 and increase of TIMP2/1 through downregulation of PI3K/AKT signaling, thus highlighting the potential role of FCL in the therapy of gastric cancer metastasis.

\section{Acknowledgements}

The present study was supported by a Youth Development Fund from the Health Authorities of Suzhou (Suzhou, China; grant no. 201017).

\section{References}

1. Qiu MZ and Xu RH: The progress of targeted therapy in advanced gastric cancer. Biomark Res 1: 32, 2013.

2. Wong $\mathrm{H}$ and Yau T: Targeted therapy in the management of advanced gastric cancer: Are we making progress in the era of personalized medicine. Oncologist 17: 346-358, 2012.

3. Murray GI, Duncan ME, Arbuckle E, Melvin WT and Fothergill JE: Matrix metalloproteinases and their inhibitors in gastric cancer. Gut 43: 791-797, 1998.

4. Pereira AL, Veras SS, Silveira EJ, Seabra FR, Pinto LP, Souza LB and Freitas RA: The role of matrix extracellular proteins and metalloproteinases in head and neck carcinomas: An updated review. Braz J Otorhinolaryngol 71: 81-86, 2005.

5. Zhang X, Wang Y, Yamamoto G and Tachikawa T: Expression of matrix metalloproteinases MMP-2, MMP-9 and their tissue inhibitors TIMP-1 and TIMP-2 in the epithelium and stroma of salivary gland pleomorphic adenomas. Histopathology 55: 250-260, 2009.
6. Desgrouas C, Taudon N, Bun SS, Baghdikian B, Bory S, Parzy D and Ollivier E: Ethnobotany, phytochemistry and pharmacology of Stephania rotunda Lour. J Ethnopharmacol 154: 537-563, 2014.

7. Fang LH, Zhang YH and Ku BS: Fangchinoline inhibited the antinociceptive effect of morphine in mice. Phytomedicine 12: 183-188, 2005.

8. Kim HS, Zhang YH, Oh KW and Ahn HY: Vasodilating and hypotensive effects of fangchinoline and tetrandrine on the rat aorta and the stroke-prone spontaneously hypertensive rat. J Ethnopharmacol 58: 117-123, 1997.

9. Lin TY, Lu CW, Tien LT, Chuang SH, Wang YR, Chang WH and Wang SJ: Fangchinoline inhibits glutamate release from rat cerebral cortex nerve terminals (synaptosomes). Neurochem Int 54: 506-512, 2009.

10. Xing Z, Zhang Y, Zhang X, Yang Y, Ma Y and Pang D: Fangchinoline induces G1 arrest in breast cancer cells through cell-cycle regulation. Phytother Res 27: 1790-1794, 2013.

11. Wang CD, Huang JG, Gao X, Li Y, Zhou SY, Yan X, Zou A, Chang JL, Wang YS, Yang GX and He GY: Fangchinoline induced G1/S arrest by modulating expression of p27, PCNA, and cyclin D in human prostate carcinoma cancer PC 3 cells and tumor xenograft. Biosci Biotechnol Biochem 74: 488-493, 2010.

12. Wang N, Pan W, Zhu M, Zhang M, Hao X, Liang G and Feng Y: Fangchinoline induces autophagic cell death via p53/sestrin2/AMPK signalling in human hepatocellular carcinoma cells. Br J Pharmacol 164: 731-742, 2011.

13. Guo B, Su J, Zhang T, Wang K and Li X: Fangchinoline as a kinase inhibitor targets FAK and suppresses FAK-mediated signaling pathway in A549. J Drug Target 23: 266-274, 2015.

14. Magee PJ, Allsopp P, Samaletdin A and Rowland IR: Daidzein, $\mathrm{R}-(+)$ equol and S-(-)equol inhibit the invasion of MDA-MB-231 breast cancer cells potentially via the down-regulation of matrix metalloproteinase-2. Eur J Nutr 53: 345-350, 2014.

15. Chen PN, Hsieh YS, Chiou HL and Chu SC: Silibinin inhibits cell invasion through inactivation of both PI3K-Akt and MAPK signaling pathways. Chem Biol Interact 156: 141-150, 2005.

16. Sampieri CL, León-Córdoba K and Remes-Troche JM: Matrix metalloproteinases and their tissue inhibitors in gastric cancer as molecular markers. J Cancer Res Ther 9: 356-363, 2013.

17. Sier CF, Kubben FJ, Ganesh S, Heerding MM, Griffioen G, Hanemaaijer R, van Krieken JH, Lamers CB and Verspaget HW: Tissue levels of matrix metalloproteinases MMP-2 and MMP-9 are related to the overall survival of patients with gastric carcinoma. Br J Cancer 74: 413-417, 1996.

18. Beliën AT, Paganetti PA and Schwab ME: Membrane-type 1 matrix metalloprotease (MT1-MMP) enables invasive migration of glioma cells in central nervous system white matter. J Cell Biol 144: 373-384, 1999.

19. Deryugina EI, Luo GX, Reisfeld RA, Bourdon MA and Strongin A: Tumor cell invasion through matrigel is regulated by activated matrix metalloproteinase-2. Anticancer Res 17: 3201-3210, 1997.

20. Chen HJ, Lin CM, Lee CY, Shih NC, Amagaya S, Lin YC and Yang JS: Phenethyl isothiocyanate suppresses EGF-stimulated SAS human oral squamous carcinoma cell invasion by targeting EGF receptor signaling. Int J Oncol 43: 629-637, 2013.

21. Lu KH, Yang HW, Su CW, Lue KH, Yang SF and Hsieh YS: Phyllanthus urinaria suppresses human osteosarcoma cell invasion and migration by transcriptionally inhibiting u-PA via ERK and Akt signaling pathways. Food Chem Toxicol 52: 193-199, 2013.

22. Yang SF, Chen MK, Hsieh YS, Yang JS, Zavras AI, Hsieh YH, Su SC, Kao TY, Chen PN and Chu SC: Antimetastatic effects of Terminalia catappa L. on oral cancer via a down-regulation of metastasis-associated proteases. Food Chem Toxicol 48: 1052-1058, 2010 